\title{
6.9 EMISSION OF GRAVITATIONAL WAVES FROM THE PULSAR
}

\section{R. RUFFINI*}

Institute for Advance Study, School of Natural Sciences, Princeton, N.J., U.S.A.

Dr. Ruffini gave a brief survey of the present experimental evidence for the existence of gravitational waves, and discussed the possibility that the shapes of neutron stars might depart from rotational symmetry, leading to the emission of gravitational waves.

The following summary has been prepared by the Editors.

The concept of gravitational waves, based on Einstein's theoretical work on General Relativity, is now widely accepted. The experimental evidence for their existence is less certain, although the experiments carried out by Weber since 1950 have given promising results. The first of these used the Earth as a detector, and gave only an upper limit for the intensity of waves with period of $54 \mathrm{~min}$. The second, using an aluminium bar resonating at $1600 \mathrm{~Hz}$, gave promising results; furthermore there are now suggested to be coincidences between times of detection at widely separated sites. It turns out that the frequency of this detector is close to the frequency of the quadrupole mode of vibration of a neutron star $\omega \sim(\pi G \varrho)^{1 / 2}$. Furthermore, the detector is directional, and there seems to be an anisotropy suggesting that the gravitational waves originate in the plane of the Galaxy.

The shape taken up by a body in very fast rotation has been studied by many people, including MacLaurin, Jacobi, Lord Kelvin, Poincare, Darwin, and Jeans. For an incompressible fluid with constant density the sequence of shapes taken up as the rotation speed increases is described by MacLaurin (flattened ellipsoids), then to a Jacobi sequence of ellipsoids in which the lengths of the three principal axes are all different, followed by pear-shaped configurations. We must investigate the possibility that a neutron star can have a Jacobi configuration, which can radiate gravitational waves. Jeans continued the analysis for a fluid with equation of state $P=k \varrho^{\gamma}$. He showed that if $\gamma>2.2$ the star can follow the Jacobi sequence, but it does not if $\gamma<2.2$.

In the Harrison-Wheeler equation of state $\gamma>2.2$ only in the region of low density, and gravitational radiation seems unlikely. In the Cameron equation of state, on the other hand, $\gamma>2.2$ for densities greater than $10^{13} \mathrm{~g} \mathrm{~cm}^{-3}$, which applies to about $90 \%$ of the star.

To give a quantitative estimate of the gravitational radiation emitted by a neutron star initially in a Jacobi ellipsoid the usual formula (linearized theory) relating the energy flux to the third derivative square of the quadrupole moment have here been used. This treatment shows the following results:

(a) A neutron star, initially in the Jacobi sequence, would decrease its angular

* Partly supported by NSF Grant GP 7669. 
momentum due to the emission of gravitational radiation and increase its angular velocity up to the encounter with the Maclaurin sequence.

(b) Typical amount of emission of gravitational radiation $\simeq 10^{52} \mathrm{ergs} / \mathrm{sec}$ should be expected in the first seconds of formation of a neutron star.

(c) An observation of this emission of gravitational radiation could give interesting indirect information on the effective $\gamma$ of the neutron star $(\gamma \geqslant 2.2)$. 